\title{
Análisis financiero del sistema de producción cafetalera en seis municipios de la provincia de Vélez (Santander, Colombia)
}

\author{
Financial analysis of the coffee production system in six \\ municipalities in the Velez province (Santander, Colombia)
}
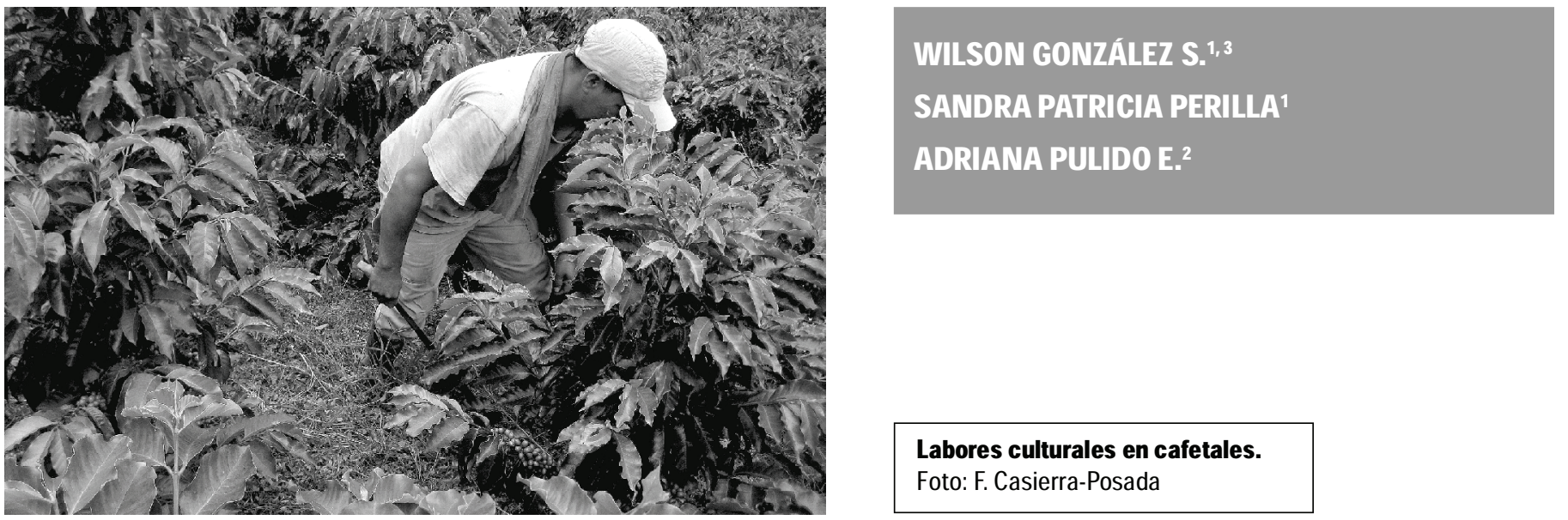

\section{RESUMEN}

La producción cafetalera en Colombia es una actividad de gran importancia; cuenta con un área sembrada de 873.000 ha y una producción anual de 12 millones de sacos de $60 \mathrm{~kg}$, y de ella dependen en forma directa cerca de dos millones de personas. En particular, el departamento de Santander participa con un 4,7\% del total de la producción nacional. Una de las falencias que se presentan en el sistema de producción del café está relacionada con el registro de los rubros implicados en su estructura de costos. En este trabajo se analizó la rentabilidad financiera del agroecosistema cafetalero en seis municipios de la provincia de Vélez, Santander. La metodología incluyó el uso de técnicas de investigación participativa y los aplicativos de Microsoft Excel denominados «TodoCostoCafe» y «Agrodinamico». Los resultados indicaron que el rubro valor de la tierra determina las diferencias en los valores de la Tasa Interna de Retorno y el Valor Presente Neto; así mismo, el asocio caféplátano-guamo aumentó la rentabilidad del agroecosistema cafetalero entre $50 \%$ y $80 \%$.

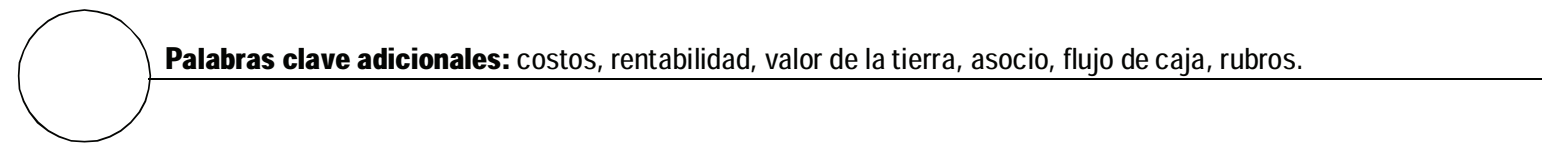

\footnotetext{
${ }^{1}$ Facultad de Ciencias Agropecuarias, Universidad Pedagógica y Tecnológica de Colombia, Tunja (Colombia)

${ }^{2}$ Comité Departamental de Cafeteros de Santander (Barbosa, Colombia)

${ }^{3}$ Autor para correspondencia: wilson.gonzalez@uptc.edu.co
} 


\section{ABSTRACT}

Coffee production in Colombia is a major activity, with a cultivated area of 873.000 ha and an annual production of 12 million $60 \mathrm{~kg}$ bags, on which close to two million people are directly dependent. In particular, the department of Santander holds $4.7 \%$ of the total national production. However, flaws can be found in the cost structure, particularly with the registration of related items. This paper analyzed the financial performance of the coffee agroecosystem in six municipalities in the Vélez Province, Santander (Colombia). The methodology included the use of participatory research techniques and applications of Microsoft Excel called «TodoCostoCafe» and «Agrodinámica.» The results indicated that the item value of land determines the differences in the values of Internal Rate of Return and Net Present Value. Furthermore, the coffee-guamo-banana association increased profitability of the coffee agroecosystem by between $50 \%$ and $80 \%$.

Additional key words: cost, effectiveness, land value, partnership, cash flow, items.

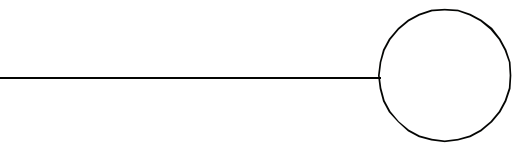

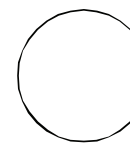

En Colombia, el cultivo del café (Coffea arabica L.), con un área sembrada de 873.000 ha y una producción anual de 12 millones de sacos de 60 $\mathrm{kg}$, se ha convertido en uno de los principales productos de la economía nacional; cerca de dos millones de personas dependen directamente de esta actividad, que se desarrolla en gran medida en fincas de pequeña y mediana extensión. En particular, el departamento de Santander participa con $4,7 \%$ del total de la producción nacional, con 42.939 ha y una producción de 470.000 sacos de 50 kg; en el 2007 el café participó con el 8,3\% del PIB del departamento (Cámara de Comercio de Bucaramanga, 2009). Específicamente, en la provincia de Vélez, la producción del grano constituye un importante renglón económico, traducido en la fuente de ingresos para aproximadamente 8.000 familias cafetaleras.

La Federación Nacional de Cafeteros de Colombia es una institución de carácter gremial, privada y sin ánimo de lucro, que tiene por objeto fomentar la caficultura colombiana, procurando el bienestar del caficultor mediante mecanismos de colaboración, participación e innovación; representa a más de 500.000 productores de café del país, lo que la ha posicionado como un importante actor institucional en Colombia; sus áreas de acción incluyen actividades y programas de carácter económico, social, científico, tecnológico, industrial y comercial, buscando mantener el carácter de capital social estratégico de la caficultura de las zonas cafeteras del país (Federación Nacional de Cafeteros de Colombia, 2010).

El servicio de extensión de la Federación Nacional de Cafeteros de Colombia es uno de los sistemas de apoyo y acompañamiento más importantes para los caficultores en este país; en especial, se encarga de la transferencia de tecnología para mejorar los distintos procesos del agroecosistema cafetalero, tales como la calidad, la productividad de la plantación y los rendimientos económicos de la finca.

Un problema sensible para los caficultores se deriva de la escasez de registros de egresos e ingresos de los distintos rubros que componen la estructura de costos del sistema de producción, que sean consensuados con los caficultores de las distintas zonas productoras del grano para su validación social; esta situación supone la inexistencia de indicadores que permitan soportar estrategias en busca de la competitividad del sector.

Uno de los programas bandera del Comité Departamental de Cafeteros de Santander es el de gestión empresarial, que, liderado por el servicio de extensión, permite fortalecer la capacidad de autogestión del agricultor para que logre un manejo eficiente de su finca y permita 
facilitar la permanencia y sostenibilidad de su negocio. En este sentido, es probable que gran parte de la caficultura tenga la posibilidad de aumentar la eficiencia productiva con mejoras en los sembrados y en la administración de las fincas.

Mora et al. (2007) destacan las bondades del análisis financiero para proponer opciones en la caficultura campesina que mejoren la rentabilidad del sistema. Este proceso es más fácil cuando existe en los caficultores la capacidad educativa y empresarial para aceptar nuevas formas de producción y gestión de los recursos, la cual no está muy difundida en las zonas cafeteras colombianas (Aguilar, 2003). El diseño de una estructura de costos como herramienta gerencial para la toma de decisiones en el agroecosistema de café permite, entre otros aspectos, racionalizar el cálculo de sus costos de producción, además de suponer una veracidad en la determinación de los precios de comercialización, y generar la posibilidad de ajustes necesarios en la optimización y racionalización en el uso de los recursos. De acuerdo con lo anterior, este proyecto analizó el sistema de producción cafetalera desde la perspectiva financiera, en seis municipios de la provincia de Vélez, Santander, buscando alternativas que motiven el mejoramiento de su competitividad.

\section{MATERIALES Y MÉTODOS}

La provincia de Vélez se encuentra ubicada en el sur del departamento de Santander; el presente trabajo realizó estudios de caso en seis de sus municipios: Vélez, Barbosa, Puente Nacional, Guavatá, Albania y Jesús María. La zona en cuestión posee una temperatura media anual de $16,7^{\circ} \mathrm{C}$ y un promedio anual de precipitaciones de $2.204 \mathrm{~mm}$, con una humedad relativa de $82,8 \%$ y un brillo solar de $1.884 \mathrm{~h}$ anual (Cenicafé, 2006).

\section{Recolección de la información}

Se utilizó un muestreo aleatorio polietápico en función de la zona y los lugares objeto de estudio, con un error permisible de $10 \%$, un nivel de confianza de $90 \%$ y una proporción del atributo que se investiga de 50\%; lo anterior resultó en
42 caficultores. Se aplicaron dos de las técnicas de diagnóstico participativo propuestas por Geilfus (1997). El flujograma de actividades permitió compilar los ítems involucrados para evaluar el proceso de producción en el sistema cafetalero. Posteriormente, con la técnica del presupuesto de cultivo, se precisaron en el tiempo dichos rubros. Fue necesario realizar un taller de consenso para estandarizar las unidades, a fin de introducir la información a los aplicativos para su análisis.

Se aplicó una entrevista focalizada, con un cuestionario conformado por cinco componentes: Iabores y prácticas de cultivo más representativas utilizadas en los cafetales tecnificados con sombrío; recolección y productividad del café; producción de almácigo; cuantificación de algunas prácticas e insumos cafeteros (pesos), y labores y prácticas utilizadas en cafetales para el control de la broca.

\section{Análisis de la información}

La determinación de los rubros se realizó en función de la experiencia de los caficultores, la cual se ajustó a los aplicativos del software Microsoft Office Excel Todocostocafe y Agrodinámico, utilizados para el análisis de los datos. Tales aplicativos se usan en el Sistema de Seguimiento Presupuestal, que estima las metas de una empresa y permite evaluar la ejecución mediante sencillos indicadores de seguimiento. Posteriormente se diseñó la matriz para la entrada de la información histórica de los costos, con el propósito de construir los distintos flujos de fondos. Finalmente, para la evaluación financiera se calculó la Tasa Interna de Retorno (TIR) y el Valor Presente Neto (VPN), en particular, definiendo los criterios para la Tasa de Interés de Oportunidad (TIO). EI VPN es la diferencia entre el valor de los beneficios y el de los costos actualizados generados por un proyecto de inversión, a una tasa de actualización determinada, que se convierte en la TIO si es la mejor alternativa, que se rechaza para evaluar dicho proyecto. La TIR es una tasa de interés presente de los ingresos y egresos esperados de una alternativa de inversión durante el horizonte de tiempo analizado (Mungaray et al., 2008).

Es importante destacar que el sistema cafetalero involucró el asocio café (Coffea arabica L.) con 
las alternativas de sombrío existentes en la zona, tales como plátano (Musa sp.) y guamo (Inga sp.).

\section{RESULTADOS Y DISCUSIÓN}

\section{Definición de la Tasa de Interés de Oportunidad}

Para el cálculo del VPN fue necesario definir el valor de la TIO; se utilizaron fundamentalmente dos criterios:

1. Los registros históricos de la rentabilidad según la agremiación

2. Los consensos, luego de realizados los talleres participativos

No obstante, es necesario precisar que los criterios de decisión de los caficultores no necesariamente corresponden a los de un inversionista financiero. De acuerdo con González (2009), para dicha decisión median otros factores, como la maximización del ingreso disponible o renta agrícola familiar. Con esta precisión se determinó, para el cálculo del VPN, una $\mathrm{TIO}$ del $10 \%$. En este sentido, este mismo valor fue el referente para la interpretación de la TIR.
Las tablas 1 y 2 muestran, para cada uno de los municipios de la zona de estudio, los flujos de caja para el horizonte de tiempo determinado, que corresponde a un ciclo de siembra y dos ciclos de zoca. Como se puede apreciar en la tabla 3 , todas las localidades presentan una TIR mayor que la TIO, como consecuencia de un ingreso neto positivo en los periodos posteriores al inicio de los ciclos. La anterior situación se soporta con Ios valores positivos resultantes del VPN. Es necesario destacar que la mayor rentabilidad financiera en función de los indicadores calculados se presenta en los municipios de Jesús María, Albania y Guavatá.

La tabla 4 muestra el valor de la hectárea de la tierra (\$) y la rentabilidad del sistema cafetalero (TIR). Si se consideran estas dos últimas variables en un escenario de aleatoriedad y se calcula su grado de asociatividad con el coeficiente de correlación $(r)$, se explica la alta relación lineal positiva de los componentes. En este sentido, un $r=0,96$ indica que en la provincia de Velez los municipios cafetaleros muestran una alta correlación entre el valor de la tierra y la rentabilidad del sistema.

Un posible contexto, en el cual se elimine el valor de la tierra, muestra que los municipios caracterizados por una baja rentabilidad inicial cambian drásticamente el valor de la TIR (tabla

\begin{tabular}{|c|c|c|c|c|c|c|c|c|c|}
\hline Item & Mun & icipio de AI & bania & Mun & cipio de $\mathrm{Ba}$ & rbosa & Mun & cipio de Gu & Iavatá \\
\hline \multirow[t]{2}{*}{ Ciclo de siembra } & Año 0 & Año 1 & Año 6 & Año 0 & Año 1 & Año 6 & Año 0 & Año 1 & Año 6 \\
\hline & & Siembra 1 & Siembra 6 & & Siembra 1 & Siembra 6 & & Siembra 1 & Siembra 6 \\
\hline Ingresos & & 0 & 8.250 .000 & & 0 & 12.000 .000 & & 0 & 8.250 .000 \\
\hline Egresos & 8.000 .000 & 3.906 .890 & 2.593 .605 & 23.000 .000 & 6.344 .902 & 4.682 .224 & 5.000 .000 & 3.792 .068 & 3.359 .856 \\
\hline Diferencia & -8.000 .000 & -3.906 .890 & 56.56 .395 & -23.000 .000 & -6.344 .902 & 7317.776 & -5.000 .000 & -3.792 .068 & 4.890 .144 \\
\hline \multirow[t]{2}{*}{\begin{tabular}{|l} 
Ciclo de primera zoca \\
\end{tabular}} & & Año 7 & Año 12 & & Año 7 & Año 12 & & Año 7 & Año 12 \\
\hline & & Zoca 1 & Zoca 6 & & Zoca 1 & Zoca 6 & & Zoca 1 & Zoca 6 \\
\hline Ingresos & & 0 & 4.500 .000 & & 0 & 6750.000 & & 0 & 5.250 .000 \\
\hline \begin{tabular}{|l|} 
Egresos \\
\end{tabular} & & 2.419 .256 & 1.432 .400 & & 4010506 & 2991348 & & 1.962 .372 & 2.238 .340 \\
\hline Diferencia & 0 & -2.419 .256 & 3.067 .600 & 0 & -4010506 & 3758652 & 0 & -1962372 & 3011660 \\
\hline \multirow[t]{2}{*}{ Ciclo de segunda zoca } & & Año 13 & Año 18 & & Año 13 & Año 18 & & Año 13 & Año 18 \\
\hline & & Zoca 1 & Zoca 6 & & Zoca 1 & Zoca 6 & & Zoca 1 & Zoca 6 \\
\hline Ingresos & & 0 & 12.500 .000 & & 0 & 29.750 .000 & & 0 & 10.250 .000 \\
\hline Egresos & & 2.419 .256 & 1.432 .400 & & 4.010 .506 & 2.991.348 & & 1.962 .372 & 2.238 .340 \\
\hline Diferencia & 0 & -2.419 .256 & 11.067 .600 & 0 & -4.010 .506 & 26.758 .652 & 0 & -1.962 .372 & 8.011 .660 \\
\hline
\end{tabular}


Tabla 2. Flujo de caja neto (\$) de la caficultura en su ciclo de vida (siembra y dos zocas). Municipios Jesús María, Puente Nacional y Vélez.

\begin{tabular}{|c|c|c|c|c|c|c|c|c|c|}
\hline \multirow{2}{*}{ Ciclo de siembra } & \multicolumn{3}{|c|}{ Municipio de Jesús María } & \multicolumn{3}{|c|}{ Municipio de Puente Nacional } & \multicolumn{3}{|c|}{ Municipio de Vélez } \\
\hline & Año 0 & Año 1 & Año 6 & Año 0 & Año 1 & Año 6 & Año 0 & Año 1 & Año 6 \\
\hline & & Siembra 1 & Siembra 6 & & Siembra 1 & Siembra 6 & & Siembra 1 & Siembra 6 \\
\hline Ingresos & & 0 & 900.0000 & & 0 & 9.000 .000 & & 0 & 9.750 .000 \\
\hline Egresos & 7.000 .000 & 3.841 .195 & 2.980 .550 & 19.000 .000 & 4.777 .805 & 3684.903 & 18.000 .000 & 4.526 .664 & 3451.185 \\
\hline Diferencia & -7000000 & -3841195 & 6019450 & -19000000 & -4777805 & 5315097 & -18000000 & -4526664 & 6298815 \\
\hline \multirow[t]{2}{*}{ Ciclo de primera zoca } & & Año 7 & Año 12 & & Año 7 & Año 12 & & Año 7 & Año 12 \\
\hline & & Zoca 1 & Zoca 6 & & Zoca 1 & Zoca 6 & & Zoca 1 & Zoca 6 \\
\hline Ingresos & & 0 & 5.250 .000 & & 0 & 6.000 .000 & & 0 & 6.750 .000 \\
\hline Egresos & & 2.432 .839 & 1.694 .000 & & 2.676 .298 & 2.531 .910 & & 2.516 .927 & 2.331 .800 \\
\hline Diferencia & 0 & -2.432 .839 & 3.556 .000 & 0 & -2.676 .298 & 3.468 .090 & 0 & -2.516 .927 & 4.418 .200 \\
\hline \multirow[t]{2}{*}{ Ciclo de segunda zoca } & & Año 13 & Año 18 & & Año 13 & Año 18 & & Año 13 & Año 18 \\
\hline & & Zoca 1 & Zoca 6 & & Zoca 1 & Zoca 6 & & Zoca 1 & Zoca 6 \\
\hline Ingresos & & 0 & 12.250 .000 & & 0 & 25.000 .000 & & 0 & 24.750 .000 \\
\hline Egresos & & 2.432 .839 & 1.694 .000 & & 2.676 .298 & 2.531 .910 & & 2.516 .927 & 2331.800 \\
\hline Diferencia & 0 & -2.432 .839 & 10.556 .000 & 0 & -2676298 & 22468090 & 0 & -2.516 .927 & 22.418 .200 \\
\hline
\end{tabular}

5). De acuerdo con Mendieta y Perdomo (2007), para los pequeños minifundistas y campesinos el valor monetario del rubro tiene relación directa e importante con sus costos totales. De esta manera, los municipios de Barbosa, Vélez y Puente Nacional quintuplican su rentabilidad inicial, y bajo este nuevo contexto se convierten en las alternativas más atractivas financieramente.

Desde la óptica de la economía campesina, un alto valor de la tierra impacta favorablemente el ingreso disponible o renta agrícola familiar, al suponer un mayor peso del valor de la producción y una disminución del pago a los factores externos (González, 2009). Con este supuesto se puede inferir que los municipios de Barbosa,

\begin{tabular}{|c|c|c|}
\hline \multirow{2}{*}{ Municipio } & \multicolumn{2}{|c|}{$\overline{\text { ITEM }}$} \\
\hline & $\operatorname{TIR}(\%)$ & VPN (10\%) (\$) \\
\hline Albania & 22,0 & 12.160 .614 \\
\hline Barbosa & 10,9 & 2.069 .130 \\
\hline Guavatá & 25,3 & 11.705 .847 \\
\hline Jesús María & 24,8 & 13.793 .628 \\
\hline Puente Nacional & 10,8 & 1.484 .055 \\
\hline Vélez & 14,6 & 8.431 .497 \\
\hline
\end{tabular}

Vélez y Puente Nacional presentan la mayor renta agrícola familiar de la zona en estudio.

Adicionalmente, se puede indicar que el valor de la productividad en el agroecosistema cafetalero no corresponde necesariamente con el costo de la tierra. Al comparar las tablas 3 y 5 se puede notar que la mayor productividad, tanto en siembra como en zoca, dada en el municipio de Barbosa, no es equivalente con el peso porcentual del valor de su tierra, respecto a los demás municipios evaluados. En los municipios de Barbosa, Vélez y Puente Nacional, la oferta de servicios en el sector rural trasciende la actividad agrícola. De acuerdo son Sanjinés et al. (2002), la presencia de un mayor número de externalidades positivas (vías de acceso, servicios públicos,

\begin{tabular}{|l|c|c|}
\hline \multicolumn{3}{|l|}{$\begin{array}{l}\text { Tabla 4. Correlación entre valor de la tierra y TIR de los } \\
\text { municipios en estudio }\left(\boldsymbol{r}_{\mathbf{x y}}=\mathbf{0 , 9 6 )} \text {. }\right.\end{array}$} \\
\hline Municipio & $\begin{array}{c}\text { Valor tierra } \\
(\mathbf{X})(\mathbf{( \$ )}\end{array}$ & $\begin{array}{c}\text { TIR } \\
(\mathbf{V})(\%)\end{array}$ \\
\hline Barbosa & 23.000 .000 & 10,9 \\
\hline Puente Nacional & 19.000 .000 & 10,8 \\
\hline Vélez & 18.000 .000 & 14,6 \\
\hline Albania & 8.000 .000 & 22,0 \\
\hline Jesús María & 7.000 .000 & 24,8 \\
\hline Guavatá & 5.000 .000 & 25,3 \\
\hline
\end{tabular}




\begin{tabular}{|c|c|c|}
\hline Municipio & $\begin{array}{l}\text { TIR sin costo } \\
\text { de tierra }(\%)\end{array}$ & $\begin{array}{c}\text { Aumento TIR } \\
(\%)\end{array}$ \\
\hline Barbosa & 49,7 & 456,0 \\
\hline Puente Nacional & 51,7 & 478,7 \\
\hline Vélez & 70,5 & 482,9 \\
\hline Albania & 60,5 & 275,0 \\
\hline Jesús María & 63,8 & 257,3 \\
\hline Guavatá & 56,3 & 222,5 \\
\hline
\end{tabular}

turismo) explicarían el comportamiento de este valor.

Este último aspecto permite deducir que actividades como el agroturismo constituyen una opción para la diversificación del espacio rural (Riveros, 2003), con lo cual se generan ingresos adicionales al cultivo y mayor oportunidad de empleo en la región; situación que implica hacer más rentable el agroecosistema del café en zonas donde los costos de la tierra son elevados y existe un alto potencial turístico, dando valor agregado a este sistema de producción y permitiendo que el inversionista tenga una fuente que genere ingreso que le permita recuperar la alta inversión inicial en el horizonte de tiempo.

Otro componente de gran importancia para la discusión es la productividad del sistema cafetalero. Al hacer un análisis de sensibilidad,
Tabla 6. Análisis de sensibilidad, con el rubro de costo pagado por arroba $(25 \mathrm{lb})$.

\begin{tabular}{|l|c|c|}
\hline \multicolumn{1}{|c|}{ Municipio } & $\begin{array}{c}\text { TIR (\%) } \$ 66.000 / \\
\text { arroba c.p.s* }\end{array}$ & $\begin{array}{c}\text { VPN } \\
(\$)\end{array}$ \\
\hline Barbosa & 7,8 & $-4.880 .979,7$ \\
\hline Vélez & 11,2 & $-4.316 .925,0$ \\
\hline Puente Nacional & 7,6 & $-4.316 .925,0$ \\
\hline Jesús María & 19,3 & $8.198 .667,2$ \\
\hline Guavatá & 18,8 & $6.366 .203,5$ \\
\hline Albania & 17,1 & $6.934 .589,1$ \\
\hline *café pergamino seco & &
\end{tabular}

tomando como valor del grano el precio de compra más bajo obtenido en el 2010 ( $\$ 6.5000$ arroba de café pergamino seco c.p.s), se observa que los municipios de Barbosa y Puente Nacional muestran una TIR menor que Ia TIO. Esta situación se puede sustentar con los valores negativos del VPN (tabla 6). El análisis de sensibilidad muestra la vulnerabilidad del sistema cafetalero en términos financieros, ante un movimiento decreciente en el precio del producto, situación que se agrava si se tiene en cuenta el exiguo crecimiento marginal de la demanda (Tobasura, 2005).

Los cuadros 7 y 8 muestran el escenario financiero con el sistema café-guamo-plátano. Los registros indican que este asocio constituye una inversión rentable que supera, para todos los municipios, el sistema cafetalero sin sombrío, especialmente en Guavatá, Jesús María y Albania

Tabla 7. Flujo de Caja Neto (\$) de la caficultora bajo sombrío en su ciclo de vida (siembra y dos zocas). Municipios Albania, Barbosa y Guavatá.

\begin{tabular}{|l|ccc|ccc|ccc|}
\hline \multicolumn{1}{|c|}{ Item } & \multicolumn{3}{|c|}{ Municipio de Albania } & \multicolumn{2}{c|}{ Municipio de Barbosa } & \multicolumn{3}{c|}{ Municipio de Guavatá } \\
\hline Ciclo de siembra & Año 0 & Año 1 & Año 6 & Año 0 & Año 1 & Año 6 & Año 0 & Año 1 & Año 6 \\
\hline & & Siembra 1 & Siembra 6 & & Siembra 1 & Siembra 6 & & Siembra 1 & Siembra 6 \\
\hline Ingresos & & 0 & 8.250 .000 & & 0 & 12.000 .000 & & 0 & 8.250 .000 \\
\hline Egresos & 8.000 .000 & 5.757 .890 & 2.659 .605 & 23.000 .000 & 8.195 .902 & 4.748 .224 & 5.000 .000 & 5.643 .068 & 3.425 .856 \\
\hline Diferencia & -8.000 .000 & -5.757 .890 & 5.590 .395 & -23.000 .000 & -8.195 .902 & 7.251 .776 & -5.000 .000 & -5.643 .068 & 4.824 .144 \\
\hline Ciclo de primera Zoca & & Año 7 & Año 12 & & Año 7 & Año 12 & Año 7 & Año 12 \\
\hline & & Zoca 1 & Zoca 6 & & Zoca 1 & Zoca 6 & & Zoca 1 & Zoca 6 \\
\hline Ingresos & & 144.000 & 4.500 .000 & & 144.000 & 6.750 .000 & & 144.000 & 5.250 .000 \\
\hline Egresos & & 2.507 .256 & 1.498 .400 & & 4.098 .506 & 3.057 .348 & & 2.050 .372 & 2.304 .340 \\
\hline Diferencia & $\mathbf{0}$ & $\mathbf{- 2 . 3 6 3 . 2 5 6}$ & $\mathbf{3 . 0 0 1 . 6 0 0}$ & $\mathbf{0}$ & $\mathbf{- 3 . 9 5 4 . 5 0 6}$ & $\mathbf{3 . 6 9 2 . 6 5 2}$ & $\mathbf{0}$ & $\mathbf{- 1 . 9 0 6 . 3 7 2}$ & $\mathbf{2 . 9 4 5 . 6 6 0}$ \\
\hline Ciclo de segunda Zoca & & Año 13 & Año 18 & & Año 13 & Año 18 & & Año 13 & Año 18 \\
\hline & & Zoca 1 & Zoca 6 & & Zoca 1 & Zoca 6 & & Zoca 1 & Zoca 6 \\
\hline Ingresos & & 144.000 & 17.300 .000 & & 144.000 & 34.550 .000 & & 144.000 & 15.050 .000 \\
\hline Egresos & & 2.507 .256 & 5.698 .400 & & 4.098 .506 & 7.191 .348 & & 2.050 .372 & 6.438 .340 \\
\hline Diferencia & $\mathbf{0}$ & -2.363 .256 & 1.1601 .600 & 0 & -3.954 .506 & 27.358 .652 & 0 & -1.906 .372 & 8.611 .660 \\
\hline
\end{tabular}




\begin{tabular}{|c|c|c|c|c|c|c|c|c|c|}
\hline \multirow{2}{*}{\begin{tabular}{|c|} 
Item \\
Ciclo de siembra \\
\end{tabular}} & \multicolumn{3}{|c|}{ Municipio de Jesús Maria } & \multicolumn{3}{|c|}{ Municipio de Puente Nacional } & \multicolumn{3}{|c|}{ Municipio de Vélez } \\
\hline & Año 0 & Año 1 & Año 6 & Año 0 & Año 1 & Año 6 & Año 0 & Año 1 & Año 6 \\
\hline & & Siembra 1 & Siembra 6 & & Siembra 1 & Siembra 6 & & Siembra 1 & Siembra 6 \\
\hline Ingresos & & & 9.000 .000 & & 0 & 9.000 .000 & & & 9750.000 \\
\hline Egresos & 7.000 .000 & 5.692 .195 & 3.046 .550 & 19.000 .000 & 6.628 .805 & 3.750 .903 & 18.000 .000 & 6.377 .664 & 3.517 .185 \\
\hline Diferencia & -7.000 .000 & -5.692 .195 & 5.953 .450 & -19.000 .000 & -6628805 & 5.249 .097 & -18.000 .000 & -6.377 .664 & 6.232 .815 \\
\hline \multirow[t]{2}{*}{ Ciclo de primera zoca } & & Año 7 & Año 12 & & Año 7 & Año 12 & & Año 7 & Año 12 \\
\hline & & Zoca 1 & Zoca 6 & & Zoca 1 & Zoca 6 & & Zoca 1 & Zoca 6 \\
\hline Ingresos & & 144.000 & 5.250 .000 & & 144.000 & 6.000 .000 & & 144.000 & 6.750 .000 \\
\hline Egresos & & 2.520 .839 & 1.760 .000 & & 2.764 .298 & 2.597 .910 & & 2.604 .927 & 2.397 .800 \\
\hline Diferencia & 0 & -2.376 .839 & 3.490 .000 & 0 & -2.620 .298 & 3.402 .090 & 0 & -2.460 .927 & 4.352 .200 \\
\hline \multirow[t]{2}{*}{ Ciclo de segunda zoca } & & Año 13 & Año 18 & & Año 13 & Año 18 & & Año 13 & Año 18 \\
\hline & & Zoca 1 & Zoca 6 & & Zoca 1 & Zoca 6 & & Zoca 1 & Zoca 6 \\
\hline Ingresos & & 144.000 & 17.050 .000 & & 144.000 & 29.800 .000 & & 144.000 & 2.9550 .000 \\
\hline Egresos & & 2.520 .839 & 5.960 .000 & & 2.764 .298 & 6.731 .910 & & 2.604 .927 & 6.531 .800 \\
\hline Diferencia & 0 & -2.376 .839 & 11.090 .000 & 0 & -2.620 .298 & 23.068 .090 & 0 & -2.460 .927 & 23.018 .200 \\
\hline
\end{tabular}

(tabla 9). Nótese que el aumento porcentual oscila entre $54 \%$ y $80 \%$. Lo anterior concuerda con Muschler (2000), quien señala que sembrar café bajo sombra significa que se pueden generar ingresos adicionales para la producción arbórea, sobre todo madera, leña y frutos.

En la región de los Tuxtlas (México) se han demostrado las bondades de la factibilidad financiera de los sistemas agroforestales (López, 2004). Por tanto, Ia diversificación de Ios agroecosistemas ha sido ampliamente reconocida como una estrategia sólida para reducir la variabilidad en precios y productividad en los cultivos e incrementar la estabilidad en el ingreso y bajar el riesgo financiero (Ramírez et al., 2001).

\section{CONCLUSIONES}

La mayor rentabilidad del sistema cafetalero en la zona de estudio se presentó en los municipios de Jesús María, Albania y Guavatá.

El valor de la tierra fue el rubro definitorio en la estructura de costos que incidió sensiblemente para la definición de la rentabilidad diferencial en los seis municipios en estudio.

Un análisis de factibilidad con un escenario en el cual se omita el valor de la tierra muestra una mayor favorabilidad de los municipios que inicialmente presentaban la más baja rentabilidad, a saber: Barbosa, Vélez y Puente Nacional.

\begin{tabular}{|l|c|}
\hline $\begin{array}{l}\text { Tabla 9. Análisis de sensibilidad incluyendo sombrío de } \\
\text { plátano y guamo. }\end{array}$ & TIR (\%) \\
\hline \multicolumn{1}{|c|}{ Municipio } & 13,5 \\
\hline Barbosa & 13,9 \\
\hline Puente Nacional & 18,30 \\
\hline Vélez & 29,90 \\
\hline Albania & 33,9 \\
\hline Jesús María & 36,7 \\
\hline Guavatá
\end{tabular}

La estructura de costos de los municipios de Barbosa y Puente Nacional explica la mayor vulnerabilidad de sus agroecosistemas cafetaleros ante una volatilidad del precio de grano. En ese sentido, el análisis de sensibilidad mostró que, con el menor precio oficial de compra de café pergamino seco del 2010, la TIR no alcanza el valor de la TIO determinada, para la valoración de la rentabilidad.

En la provincia de Vélez, el sistema cafetalero café-guamo-plátano es factible desde la perspectiva financiera, al presentar tasas de rentabilidad superiores al interés de oportunidad calculado para dicha zona. 


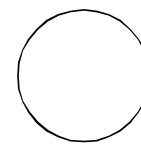

AGRADECIMIENTOS

Los autores expresan su agradecimiento al Comité Departamental de Cafeteros de Santander, en particular, al Programa de Gestión Empresarial.

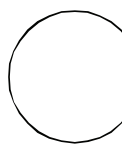

REFERENCIAS BIBLIOGRÁFICAS

Aguilar, L. 2003. Crisis del café y el desarrollo regional. Cuadernos de Economía (22)38, 239-272.

Cámara de Comercio de Bucaramanga. 2009. Indicadores económicos. En: http: //www.camaradirecta.com; consulta: mayo de 2010.

Cenicafé. 2006. Anuario meteorológico cafetero. En: Centro Nacional de Investigación de café. En: http: www.cenicafe.org. 564 p.; consulta: marzo de 2010.

Federación Nacional de Cafeteros de Colombia. 2010. Sistema de Información Cafetera. Encuesta Nacional Cafetera SICA. Estadísticas Cafeteras. Disponible online en: http://www.federacion decafeteros.org/caficultores/es/ s e r vi cios_para_e I_cafetero/ sistema_de_informacion_sica; consulta: octubre de 2010.

Geilfus, F. 1997. 80 herramientas para el desarrollo participativo: Diagnóstico, planificación, monitoreo y evaluación. Instituto Interamericano de Cooperación para la Agricultura, San Salvador, EI Salvador.

González, W. 2009. Educación y entorno en la Ingeniería Agronómica. Facultad de Ciencias Agropecuarias y Facultad de Ciencias Económicas y Administrativas, UPTC, Tunja, Colombia.

López, E. 2004. Manual para el diseño, establecimiento y manejo de los principales sistemas agroforestales como cedro rojo, cedro nogal y primavera en los Tuxtlas, Veracruz. Tesis. Universidad Autónoma de Chapingo, Chapingo, México.
Mendieta, J. y J. Perdomo. 2007. Factores que afectan la eficiencia técnica y asignatura en el sector cafetero colombiano: una aplicación con análisis envolvente de datos. Desarrollo y Sociedad 60, 1-25.

Mora, J.; C. Ramírez y O. Quiros. 2007. Labor, costbenefit analysis, and energy productivity in peasant coffee growing farms in Puriscal, Costa Rica. Cuadernos de Administración (20)33, 49-76.

Mungaray, A.; F. Castellanos y M. Sánchez. 2008. Asistencia microempresarial a través del servicio social universitario. Asociación Nacional de Universidades e Instituciones de Educación superior (ANUIES), México.

Muschler R. 2000. Árboles en cafetales. Colección módulos de enseñanza agroforestal. Centro Agronómico Tropical de Investigación y Enseñanza (CATIE), Turrialba, Costa Rica.

Ramírez, O.; E. Somarriba; T. Ludewigs y P. Ferreira. 2001. Financial returns, stability and risk of cacaoplantain-timber agroforestry systems in Central America. Agroforestry Systems 51, 141-154.

Riveros, H. 2003. El agroturismo, una alternativa para revalorizar la agroindustria rural como mecanismo de desarrollo local. Serie de documentos de trabajo, Prodar, Lima.

Sanjinés, F.; B. Vargas y Z. Herrera. 2002. Ventajas comparativas y competitivas del comercio regional orureño. Offset Boliviana Ltda., La Paz.

Tobasura, I. 2005. La crisis cafetera: una oportunidad para el cambio en las regiones cafeteras de Colombia. Agronomía 13(2), 35-46. 